04

\title{
Ионно-электронная рекомбинация и тепловые потоки в высокочастотных ионных двигателях
}

\author{
(C) В.К. Абгарян ${ }^{1}$, В.Ю. Гидаспов ${ }^{2}$, А.Б. Надирадзе ${ }^{2}$, А.А. Семенов ${ }^{2}$ \\ ${ }^{1}$ Научно-исследовательский институт прикладной механики и электродинамики \\ Московского авиационного института (Национального исследовательского университета), Москва, Россия \\ ${ }^{2}$ Московский авиационный институт (Национальный исследовательский университет) (МАИ), Москва, Россия \\ E-mail: vka.mai@mail.ru
}

Поступило в Редакцию 30 октября 2018 г.

В окончательной редакции 30 октября 2018 г.

Принято к публикации 15 ноября 2018г.

Рассмотрены тепловые потоки, идущие из плазмы на поверхности элементов конструкции высокочастотных ионных двигателей с перфорированными электродами ионно-оптической системы. Лучистый поток, образуемый при ионно-электронной рекомбинации на поверхностях, граничащих с плазмой, рассматривается с учетом пространственного распределения и частичного отражения излучения от поверхностей. Приведены новые выражения для тепловых потоков, которые следует использовать в качестве граничных условий в численных моделях расчета температур в высокочастотных ионных двигателях.

DOI: 10.21883/PJTF.2019.04.47325.17576

Высокочастотные ионные двигатели (ВЧИД) применяются в космических исследованиях с конца прошлого столетия. Высокая скорость истечения ионов рабочего газа (десятки $\mathrm{km} / \mathrm{s}$ ) позволяет использовать ионные двигатели, в том числе и ВЧИД, для стабилизации орбит и ориентации космических аппаратов. Конструкция ВЧИД была предложена Лёбом [1]. Им же описаны физические процессы, протекающие в ВЧИД. Типичная схема ВЧИД изображена на рис. 1. Основными узлами конструкции являются керамическая газоразрядная камера (ГРК); индуктор, выполненный в виде медной спирали, подключенный к высокочастотному (ВЧ) генератору; ионнооптическая система (ИОС), состоящая из эмиссионного и ускоряющего электродов (ЭЭ и УЭ), перфорированных соосными отверстиями; газоввод, через который рабочий газ подается в газоразрядную камеру; катоднейтрализатор, испускающий электроны для компенсации положительного заряда ионов, выходящих из ВЧИД, при формировании квазинейтрального потока.

В процессе работы двигателя происходит нагрев элементов его конструкции компонентами плазмы, который может влиять на работу двигателя. Поэтому для определения теплового состояния двигателей обычно выполняется численный расчет температур. В расчетных моделях в качестве граничных условий используются тепловые потоки, выносимые из разряда компонентами плазмы: ионами, электронами и фотонами.

Выражения для тепловых потоков были впервые приведены Ван Ноордом [2] для ионных двигателей Кауфмана на постоянном токе и использованы в разработанной им же расчетной модели [3]. С небольшими отличиями такие же выражения для тепловых потоков используются для ВЧИД во всех известных расчетных моделях [4-6].

Схема тепловых потоков для ВЧИД изображена на рис. 2. Наибольшими по величине являются тепловые потоки, образующиеся за счет выпадения на стенки ионов и электронов из плазмы. Заметная часть тепловой мощности приносится на стенки фотонами, образующимися за счет потери части входной ВЧ-мощности на возбуждения атомов и ионов рабочего газа, не приводящие к ионизации и снимаемые излучением квантов света. В ВЧИД эта часть составляет около $15 \%$ от входной ВЧ-мощности $W_{\mathrm{HF}}$. Наиболее распространенные в расчетных моделях выражения для тепловых потоков приведены в [6] в интегральной форме. Они имеют следующий вид:

$$
\begin{gathered}
W_{\mathrm{GDC}}=I_{\mathrm{GDC}}\left(\Delta U+\frac{5}{2} T_{e}+V_{i}\right) \\
W_{E E}=I_{i \mathrm{EE}}\left(\Delta U+\frac{1}{2} T_{e}+V_{i}-\varphi_{e \mathrm{EE}}\right)+I_{e \mathrm{EE}}\left(\varphi_{e}+2 T_{e}\right), \\
W_{\mathrm{CEX}}=I_{\mathrm{CEX}}\left(U^{-}+V_{i}-\varphi_{e \mathrm{AE}}\right),
\end{gathered}
$$

где $W_{\mathrm{GDC}}$ и $W_{\mathrm{EE}}-$ мощности, приносимые ионами и электронами на поверхность ГРК и ЭЭ соответственно; $W_{\mathrm{CEX}}$ - мощность, приносимая ионами перезарядки (charge-exchange ions) на ускоряющий электрод; $I_{\mathrm{GDC}}$, $I_{i \mathrm{EE}}, I_{e \mathrm{EE}}-$ ионный ток, падающий на поверхность ГРК, а также ионный и электронный токи, поступающие на ЭЭ, соответственно; $I_{\text {CЕХ }}$ - ток ионов перезарядки, идущий на ускоряющий электрод и составляющий 1-2\% от ионного тока $I_{0}$, выходящего из двигателя; $V_{i}-$ потенциал ионизации атомов рабочего газа; $\Delta U-$ пристеночное падение потенциала плазмы вблизи поверхностей ГРК и ЭЭ; $T_{e}$ - температура электронов, измеряемая в вольтах; $U^{-}$- потенциал ускоряющего

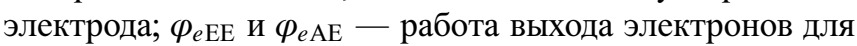
материала ЭЭ и УЭ соответственно, также выраженная в вольтах. 


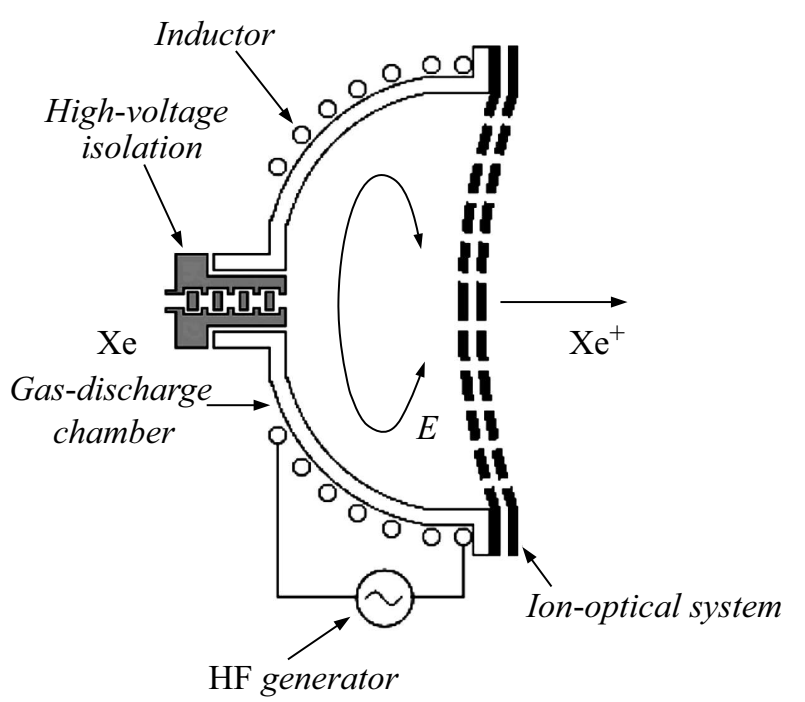

Рис. 1. Схема конструкции высокочастотного источника ионов.

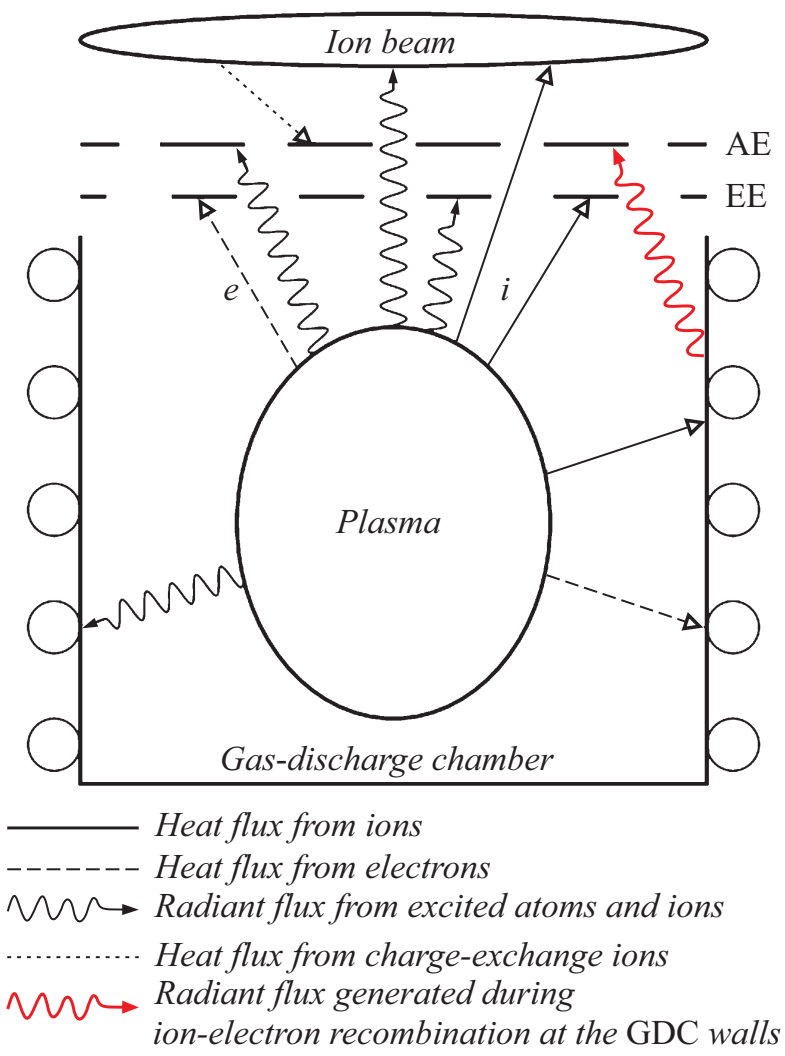

Рис. 2. Схема тепловых потоков, идущих из плазмы разряда [6].

Ионы перезарядки образуются в объеме струи выходящего из ВЧИД ионного потока за счет резонансной перезарядки ионов основного потока на нейтральных атомах рабочего газа. Двигаясь по электростатическому полю, ионы могут попасть на УЭ, находящийся под отрицательным потенциалом. Этот механизм переноса тепла на ускоряющий электрод давно и хорошо известен.
Для определения суммарных значений тепловых потоков, поступающих на ГРК и электроды ИОС, к выражениям (1)-(3) добавляется поток тепловой мощности $W^{*}$, приносимый фотонами, образующимися при снятии возбуждения атомов и ионов рабочего газа. Данный поток в моделях равномерно распределяется по поверхностям ГРК и ЭЭ. Часть его через отверстия в ИОС попадает на ускоряющий электрод.

Один из тепловых потоков в ВЧИД образуется при рекомбинации ионов и электронов из плазмы на поверхностях, граничащих с разрядом. Этот поток отображается в выражениях (1)-(3) слагаемым $V_{i}$. Однако такой вид слагаемого означает, что вся выделенная энергия ионизации $e V_{i}$ считается поглощенной поверхностью именно в той точке, где рекомбинация произошла. Это не соответствует физической картине процесса ионноэлектронной рекомбинации. Образующиеся в результате рекомбинации фотоны имеют, очевидно, распределение по пространству, близкое, скорее, к изотропному. И только половина фотонов, имеющая проекцию скорости, направленную к поверхности, может быть поглощена стенкой. Оставшаяся половина фотонов уходит в окружающее пространство. Во всех тепловых моделях не принимается во внимание тот факт, что рекомбинационное излучение может частично отражаться от поверхностей. Также не учитывается то, что часть излучения неизбежно уходит через отверстия в эмиссионном электроде, прозрачность которого в подавляющем большинстве используемых двигателей близка к 0.5. Все это приводит к систематической погрешности определения суммарных тепловых потоков, поступающих на поверхности в ВЧИД. В (1) и (2) вместо слагаемого $V_{i}$ нужно использовать выражения $\alpha_{\mathrm{GDC}} V_{i}$ и $\alpha_{\mathrm{EE}} V_{i}$ соответственно, где коэффициенты $\alpha_{\mathrm{GDC}}$ и $\alpha_{\mathrm{EE}}$ следует рассчитывать с учетом изложенных выше обстоятельств. Оценки показывают, что $\alpha_{\mathrm{GDC}}<1$ и $\alpha_{\mathrm{EE}}<1$, что означает уменьшение тепловых потоков от рекомбинационного излучения, поступающих на поверхности ГРК и ЭЭ. Поток тепловой мощности от рекомбинационного излучения $W_{\mathrm{AE}}^{\mathrm{REC}}$, поступающий на УЭ, не учтен ни в одной модели. Его следует добавить, и тогда суммарный тепловой поток на УЭ представляется в виде суммы не двух, а трех компонент:

$$
W_{\mathrm{AE}}=W_{\mathrm{CEX}}+W_{\mathrm{AE}}^{*}+W_{\mathrm{AE}}^{\mathrm{REC}},
$$

причем в отличие от (3) поток мощности $W_{\text {СЕХ }}$ следует использовать в виде, учитывающем также и пространственное распределение излучения, а именно

$$
W_{\mathrm{CEX}}=I_{\mathrm{AE}}\left(U^{-}+\frac{1}{2}\left(1-R_{\mathrm{AE}}\right) V_{i}-\varphi_{e \mathrm{AE}}\right),
$$

где $R_{\mathrm{AE}}-$ коэффициент отражения рекомбинационного излучения от материала УЭ.

Множитель 1/2 во втором слагаемом (5) как раз и означает уход части излучения в пространство вне двигателя. 
Были выполнены оценочные расчеты с целью определения величины изменений тепловых потоков при использовании предлагаемого в работе подхода по сравнению со значениями тепловых потоков, рассчитываемыми исходя из (1)-(3). Расчеты выполнялись для схемы ВЧИД с полусферической ГРК. В качестве материалов были выбраны керамика $\mathrm{Al}_{2} \mathrm{O}_{3}$ для ГРК, а также Ті и $\mathrm{C}$ для материалов электродов ИОС. Значения коэффициентов отражения $R_{\mathrm{GDC}}, R_{\mathrm{EE}}$ и $R_{\mathrm{AE}}$ материалов в области ультрафиолетового излучения были взяты из работ $[7,8]$. Расчеты показали, что суммарная тепловая мощность, приходящая на поверхности ГРК и ЭЭ, уменьшается незначительно (примерно на 3-5\%). В то же время тепловая мощность, поглощаемая ускоряющим электродом, увеличивается на $50 \%$, что приводит к существенному увеличению рассчитываемых температур УЭ.

Таким образом, в качестве граничных условий в численных моделях расчета температур в ионных двигателях, в которых электроды ионно-оптических систем выполнены в перфорированном отверстиями виде, для тепловых потоков следует использовать скорректированные выражения, в которых рекомбинационное излучение вводится в рассмотрение в более общем виде.

Работа выполнена при финансовой поддержке Министерства образования и науки РФ в рамках государственного задания (проект № 9.9055.2017/БЧ).

\section{Список литературы}

[1] Loeb $H$. State of the art and recent developments of the radio frequency ion motors // AIAA 7th Electric Propulsion Conf. Williamsburg, USA, 1969. P. 285.

[2] Van Noord J.L. Thermal modeling of an ion thruster. PhD Dissertation. University of Michigan, 1999. $140 \mathrm{p}$.

[3] Van Noord J.L. NEXT ion thruster thermal model // 43rd AIAA Joint Propulsion Conf. Cincinnati, 2007. AIAA Paper 2007-5218.

[4] Waldemar G., Lotz B., Meyer B.K. 3D thermal simulation of a $\mu$ N-RIT // 33rd Int. Electric Propulsion Conf. The George Washington University, USA, 2013. IEPC-2013-298.

[5] Abgaryan V.K., Kruglov K.I. // J. Surf. Invest.: X-Ray, Synchrotron Neutron Tech. 2015. V. 9. P. 1137-1143.

[6] Dobkevicius M., Feili D. // Eur. Phys. J. D. 2016. V. 70. P. 227.

[7] Polyanskiy M.N. Refractive index database. Available at: https://refractiveindex.info (accessed 23.04.2018).

[8] French R.H., Müllejans H., Jones D.J. // J. Am. Ceram. Soc. 1998. V. 81. N 10. P. 2549-2557. 